\title{
THE EFFECT OF SYNTHETIC OCTACALCIUM PHOSPHATE IN A COLLAGEN SCAFFOLD ON THE OSTEOGENICITY OF MESENCHYMAL STEM CELLS
}

\author{
Tadashi Kawai ${ }^{1,2, \S}$, Takahisa Anada ${ }^{2,}$, Taisuke Masuda $^{3}$, Yoshitomo Honda ${ }^{2}$, Yuhiro Sakai ${ }^{4,5}$, Yukio Kato \\ Shinji Kamakura ${ }^{6}$, Seishi Echigo ${ }^{1}$ and Osamu Suzuki ${ }^{2, *}$ \\ ${ }^{1}$ Division of Oral Surgery, Tohoku University Graduate School of Dentistry \\ ${ }^{2}$ Division of Craniofacial Function Engineering, Tohoku University Graduate School of Dentistry \\ ${ }^{3}$ Department of Micro-Nano Systems Engineering, Graduate School of Engineering, Nagoya University \\ ${ }^{4}$ Department of Dental and Medical Biochemistry, Hiroshima University Graduate School of Biomedical Sciences \\ ${ }^{5} \mathrm{GC}$ Corporation \\ ${ }^{6}$ Bone Regenerative Engineering Laboratory, Graduate School of Biomedical Engineering, Tohoku University
}

$\S$ These two authors contributed equally to this work

\begin{abstract}
Although the efficacy of the in vivo osteogenic capabilities of synthetic octacalcium phosphate (OCP) crystal implantation can be explained through its stimulatory capacity for the differentiation of the host osteoblastic cell lineage, direct evidence that OCP supports bone regeneration by osteogenic cells in vivo has not been shown. Mesenchymal stem cells (MSCs) isolated from 4-week-old male Wistar rat long bones were pre-incubated in osteogenic or maintenance medium in the presence or absence of basic fibroblast growth factor (bFGF). OCP/Collagen (OCP/Col) or collagen disks were seeded with MSCs that had been pre-incubated in osteogenic medium containing bFGF, which exhibited the highest differentiation induction, and then incubated for an additional day. The disks were implanted in critical-sized calvaria defects of 12-weekold male Wistar rats and the specimens were analysed radiographically, histologically, histomorphometrically, and by micro-computed tomography (CT) imaging at 4 and 8 weeks after the implantation. The $\mathrm{OCP} / \mathrm{Col} \cdot \mathrm{MSCs}$ group rapidly induced more bone regeneration, even within 4 weeks, compared to the $\mathrm{OCP} / \mathrm{Col}$ group without MSCs. The bone mineral density of the OCP/Col-MSCs group was also greater than the $\mathrm{OCP} / \mathrm{Col}$ group. The $\mathrm{Col} \cdot \mathrm{MSCs}$ group did not exhibit prominent osteogenicity. These results indicate that OCP crystals in a collagen matrix efficiently promote exogenously introduced osteogenic cells to initiate bone regeneration if the cells are pre-treated in a suitable differentiation condition.
\end{abstract}

Keywords: Octacalcium phosphate, collagen, mesenchymal stem cells, basic fibroblast growth factor, bone regeneration.

*Address for correspondence:

Osamu Suzuki

Division of Craniofacial Function Engineering

Tohoku University Graduate School of Dentistry

4-1 Seiryo-machi, Aoba-ku, Sendai 980-8575, Japan

Telephone Number: +81-22-717-7635

FAX: +81-22-717-7637

E-mail: suzuki-o@m.tohoku.ac.jp

\section{Introduction}

In the field of tissue engineering, the number of basic and clinically related studies on the use of bone marrow stromal cells (BMSCs) for the repair of large bone defects without autologous bone transplantation has increased remarkably (Kawate et al., 2006; Kuznetsov et al., 2008). BMSCs can provide a source of mesenchymal stem cells (MSCs) that can differentiate into osteoblastic cells (Dennis et al., 2007). MSCs incubated at suitable passage exhibit an excellent ability to implant at bone defect sites in vivo and could potentially replace autologous bone transplantation as a therapeutic intervention (Quarto et al., 2001). In addition, it is becoming clear that the osteogenic ability of MSCs can be combined with suitable scaffold materials for in vivo bone regeneration (Grundel et al., 1991; Werntz et al., 1996).

Calcium phosphate ceramics, such as hydroxyapatite (HA; $\left.\mathrm{Ca}_{10}\left(\mathrm{PO}_{4}\right)_{6}(\mathrm{OH})_{2}\right)$ and $\beta$-tricalcium phosphate $\left(\beta\right.$-TCP; $\left.\mathrm{Ca}_{3}\left(\mathrm{PO}_{4}\right)_{2}\right)$, have prominent osteoconductive properties and therefore have been used individually as bone substitute materials to assist in bone regeneration (Bucholz, 2002; LeGeros, 2002). These calcium phosphate ceramics have also been implanted together with MSCs in order to enhance bone regeneration in cases of severe bone atrophy or large bone defects where self-regeneration cannot occur. Calcium phosphate has been found to affect MSC functions in both in vitro and in vivo studies. Moreover, porous HA blocks have been shown to function as a scaffold when implanted in the subcutaneous tissues of nude mice together with human MSCs derived from bone marrow, even when the cells were obtained from patients with various bone-related diseases (Yoshikawa et al., 2004). Ca-deficient $\mathrm{HA}\left(\mathrm{Ca}_{10-\mathrm{X}} \mathrm{H}_{\mathrm{X}}\left(\mathrm{PO}_{4}\right)_{6}(\mathrm{OH})_{2-\mathrm{X}}, 0 \leq \mathrm{X}\right.$ $\leq 2$ ) (Winand, 1965) can support human MSC-mediated bone induction at ectopic sites (Kasten et al., 2005). In addition, biphasic calcium phosphate (BCP) composed of HA and $\beta$-TCP has been shown to provide spaces for MSC interaction and displays bone-inducing abilities in various animal species, including mouse (Chevallier et al., 2010), rat (Espitalier et al., 2009), and dog (Kuznetsov et al., 2008).

Synthetic octacalcium phosphate (OCP: $\mathrm{Ca}_{8} \mathrm{H}_{2}\left(\mathrm{PO}_{4}\right)_{6}$ - $\left.5 \mathrm{H}_{2} \mathrm{O}\right)$ is another potential scaffold for MSCs, because OCP and OCP-polymer complexes, such as OCP/ Collagen $(\mathrm{OCP} / \mathrm{Col})$, enhance bone regeneration and 
tend to be replaced with newly formed bone if implanted in experimentally created bone defects or if introduced as onlay grafts on cortical bone in various animal models (Suzuki et al., 1991; Sugihara et al., 1995; Sugihara et al., 1996; Kamakura et al., 1999; Kamakura et al., 2006; Kawai et al., 2009). OCP is known to be a potential bone apatite precursor phase (Brown et al., 1962) and is more soluble than HA or $\beta$-TCP at a physiological $\mathrm{pH}$ (Brown et al., 1981; Chow, 2001). Although the composition of biological tissue fluid is considered to be almost saturated with respect to OCP crystals (Driessens et al., 1978; Eidelman et al., 1987), OCP tends to convert to HA (Cadeficient HA) during bone regeneration in vivo (Suzuki et al., 1991; Suzuki et al., 2006b). This conversion occurs because OCP is composed of apatite layers stacked together with hydrated layers, and has a triclinic crystal system that can be converted to HA by hydrolysis of the hydrated layers in OCP (Brown et al., 1962). OCP is converted into HA through (1) a dissolution-reprecipitation mechanism and (2) topotaxial conversion by ion diffusion within the crystal lattice (Brown et al., 1981), which consumes calcium ions from the surrounding milieu and releases phosphate ions from the crystals (Brown et al., 1981; Suzuki et al., 2006a). The capacity of OCP to enhance bone regeneration could be associated with its ability to activate host osteoblasts and induce their acquisition through cell surface interactions with surrounding cells, tissue fluid, and serum proteins (Suzuki et al., 1993), which is caused by the OCP-HA conversion process (Suzuki et al., 2006b). OCP supports osteoblastic cell differentiation (Suzuki et al., 2006b) and has been shown to increase the in vitro expression of mRNA of associated genes, such as alkaline phosphatase (ALP) and osterix (Osx), in a dose-dependent manner (Anada et al., 2008). OCP also increases the ALP activity of host osteoblastic cells in vivo (Kikawa et al., 2009; Miyatake et al., 2009). Based on the experimental evidence to date, we hypothesised that OCP may promote MSCs to initiate bone regeneration around the implanted OCP, which has been observed with host osteoblastic cells. To date, the question of whether OCP can induce bone regeneration in vivo when implanted with MSCs prepared using tissue engineering techniques has not been examined.

The aim of the present study was to investigate whether seeding an OCP/Col composite with MSCs induces better bone formation than a composite without MSCs when implanted in critical-sized calvaria defects in rats.

\section{Materials and Methods}

\section{Preparation of octacalcium phosphate, collagen disks, and OCP/Col disks}

OCP was prepared by mixing calcium and phosphate solutions as previously described (Suzuki et al., 1991). Sieved OCP granules (particle sizes 300-500 $\mu \mathrm{m}$ ) obtained from the dried OCP were sterilised by heating them at $120{ }^{\circ} \mathrm{C}$ for $2 \mathrm{~h}$. As shown in our previous study, this heating technique does not affect the physical properties, such as the crystalline structure or specific surface area of the OCP granules (Suzuki et al., 1993; Suzuki et al., 1995), although it has been reported that increasing the temperature above $100{ }^{\circ} \mathrm{C}$ can induce the collapse of the OCP structure due to dehydration (Fowler et al., 1966; Nelson and McLean, 1984). A $1 \%$ solution of collagen was purchased (NMP collagen PS; Nippon Meat Packers, Tsukuba, Ibaraki, Japan) and adjusted to $\mathrm{pH} 7.4$ in order to form a gel of collagen fibrils, which was then condensed by centrifugation. A $3 \%$ collagen suspension was then prepared from the condensed collagen suspension. OCP granules with a $300-500 \mu \mathrm{m}$ diameter were added to the concentrated collagen and mixed. The weight percentage of OCP in the OCP/Col mixture was $77 \%$. The mixture was then lyophilised, and disks were moulded to a $9 \mathrm{~mm}$ diameter and $1 \mathrm{~mm}$ thickness. The moulded OCP/Col and collagen disks were subjected to a dehydrothermal treatment at $150{ }^{\circ} \mathrm{C}$ for $24 \mathrm{~h}$ in a vacuum drying oven (DP32, Yamato Scientific, Tokyo, Japan) and were then sterilised using electron beam irradiation ( $5 \mathrm{kGy}$ ). The $\mathrm{OCP} / \mathrm{Col}$ disk had a relatively small pore size of $48 \mu \mathrm{m}$. The porosity was evaluated by mercury intrusion and determined to be $92 \%$, and the elastic modulus was 0.4 $\mathrm{MPa}$, as estimated by mechanical testing (Suzuki et al., 2009; Matsui et al., 2010).

\section{Isolation and culture of MSCs}

MSCs were isolated from the bone shaft of femurs and tibias of 4-week-old male Wistar rats (SLC, Hamamatsu, Shizuoka, Japan). After both ends of the femurs or tibias were removed from the epiphysis, the bone marrow was flushed out with $10 \mathrm{~mL}$ of maintenance medium consisting of Dulbecco's modified Eagle medium (DMEM) supplemented with $10 \%$ foetal bovine serum (FBS) and $1 \%$ penicillin/streptomycin (Invitrogen-Gibco, Carlsbad, CA, USA) through a syringe with a 25 -gauge needle. The cells were maintained in a humidified atmosphere of 95 $\%$ air with $5 \% \mathrm{CO}_{2}$ at $37{ }^{\circ} \mathrm{C}$. As a separate method, the MSCs were also isolated with an osteogenic differentiation medium consisting of DMEM with $10 \%$ FBS, $1 \%$ penicillin/streptomycin, $10^{-7} \mathrm{M}$ dexamethasone, $10 \mathrm{mM}$ $\beta$-glycerophosphate, $50 \mu \mathrm{g} / \mathrm{mL}$ ascorbate 2-phosphate, and $2 \mathrm{mM}$ L-glutamine (MSCs isolated with osteogenic differentiation medium; osteogenic MSCs). The MSCs were maintained in $10 \mathrm{~mL}$ of medium at $37^{\circ} \mathrm{C}$ in a $5 \%$ $\mathrm{CO}_{2}$ environment and were detached from the dish 3-4 d after the cell isolation.

\section{Measurement of proliferation and alkaline phosphatase (ALP) activity of MSCs}

MSCs $\left(3 \times 10^{4}\right)$ were suspended in $250 \mu \mathrm{L}$ of maintenance medium and seeded in a 48 -well tissue culture plate. The MSCs were cultured in maintenance medium containing $3 \mathrm{ng} / \mathrm{mL}$ of basic fibroblast growth factor (bFGF) for 7 $\mathrm{d}$, which was almost $10 \mathrm{~d}$ after MSC isolation. After this culturing period, the medium was changed to an osteogenic medium. This culture process is referred to as the "normal method" (Fig. 1AIII). Osteogenic MSCs $\left(3 \times 10^{4}\right)$ were then suspended in $250 \mu \mathrm{L}$ of osteogenic medium and seeded in a 48 -well tissue culture plate. The osteogenic MSCs were cultured in osteogenic medium with bFGF (osteogenic (FGF+)) or without bFGF (osteogenic (FGF)) (Fig. 1AI, II). For prolonged incubation, the culture medium was exchanged with fresh medium every $3 \mathrm{~d}$. 
(A) MSCs culture for in vitro assays

(I) osteogenic (FGF+)

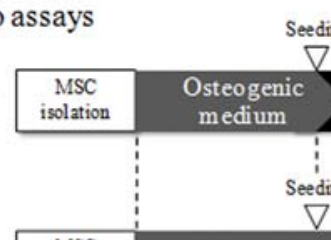

(II) osteogenic (FGF-)

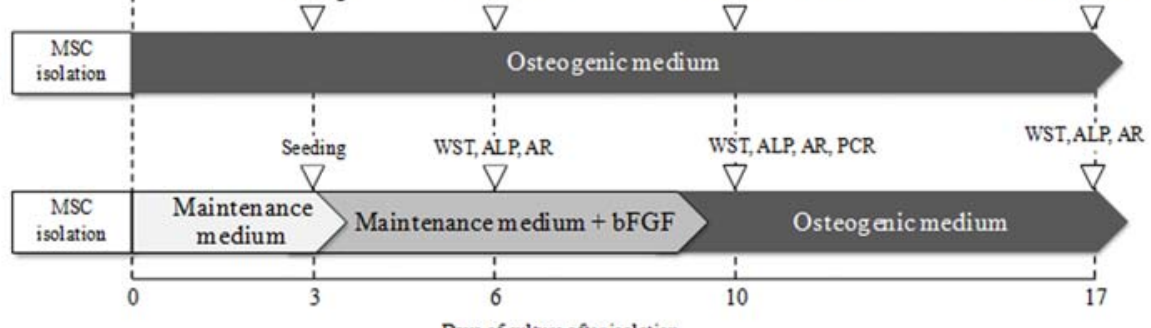

(B) MSCs preparation for in vivo assays

Days of culture after isolation

(I) osteogenic $(\mathrm{FGF}+)$

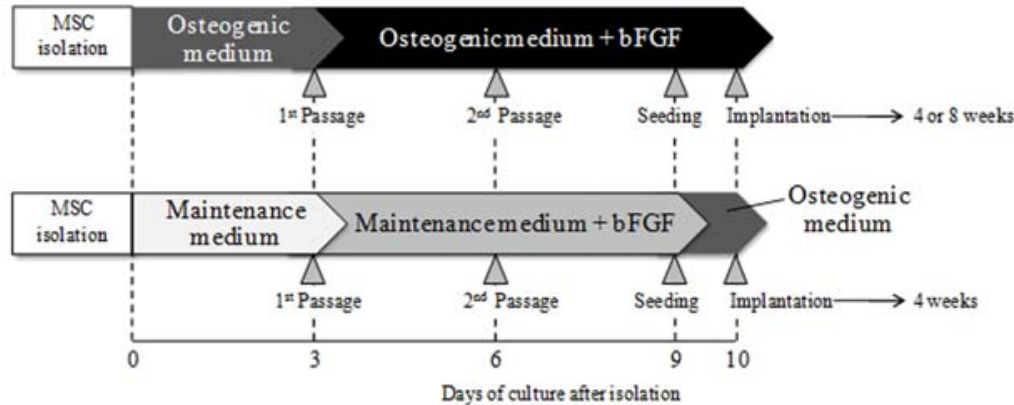

Fig. 1. Cell culture conditions of the present study. (A) MSCs culture for in vitro assays (I) MSCs were cultured in osteogenic medium from MSC isolation and with bFGF at $3 \mathrm{~d}$ (osteogenic (FGF+)). (II) MSCs were cultured in osteogenic medium from MSC isolation without bFGF (osteogenic (FGF-)). (III) As the normal method, MSCs were cultured in maintenance medium and with bFGF at $3 \mathrm{~d}$ for MSC proliferation, and osteogenic medium was used from $9 \mathrm{~d}$. WST: cell proliferation measured by WST-8 assay, ALP: cell differentiation measured by ALP activity, AR: the formation of bone nodule measured by Alizarin Red staining, PCR: cell differentiation measured by TaqMan realtime PCR. (B) MSCs culture for in vivo assays. (I), (II) MSCs were cultured with the same method as osteogenic (FGF+) (Fig. 1A(I)) and normal (Fig.1 A(III)), respectively, until 9 d. At 9 d, MSCs were seeded on OCP/Collagen disks or collagen disks. After $1 \mathrm{~d}$ culture, MSCs with OCP/Collagen disks or collagen disks were implanted in rat calvaria bone defect.

The cell number of normal MSCs, osteogenic (FGF+) MSCs, and osteogenic (FGF-) MSCs in the plates was determined at 3, 7 and $14 \mathrm{~d}(6,10$ and $17 \mathrm{~d}$ after MSC isolation, respectively) by counting living cells using a WST-8 assay (Cell Counting Kit-8, Dojindo, Kumamoto, Japan). ALP activity was measured using a commercially available labAssay ${ }^{\mathrm{TM}}$ ALP kit (Wako Pure Chemical Industries, Osaka, Japan). Briefly, on days 3, 7 and 14 of the culture period $(6,10$ and $17 \mathrm{~d}$ after MSC isolation, respectively), normal MSCs, osteogenic (FGF+) MSCs, and osteogenic (FGF-) MSCs were lysed in $0.2 \%$ Triton $\mathrm{X}-100$ solution at room temperature. The lysates were subjected to ultrasonication and centrifugation $(5000 \mathrm{~g}$ for $5 \mathrm{~min}$ ). The supernatants were then collected and the ALP activity was determined by measuring the conversion of p-nitrophenol to p-nitrophenylate at $\mathrm{pH} 9.8$ and $37^{\circ} \mathrm{C}$ for $30 \mathrm{~min}$. The ALP activity was normalised using the cell number that was determined by the WST- 8 assay.

\section{Alizarin red staining of MSCs}

On days 3, 7 and 14 of the culture period (6, 10 and 17 $\mathrm{d}$ after MSC isolation, respectively), the monolayer of normal, osteogenic (FGF+), and osteogenic (FGF-) MSCs was washed twice with phosphate-buffered saline (PBS), rinsed with water, and stained with alizarin red S (Wako
Pure Chemical Industries) for $30 \mathrm{~min}$ at room temperature. After staining, the cells were rinsed with water and dehydrated in 70 to $100 \%$ ethanol.

\section{TaqMan quantitative real-time polymerase chain reaction}

After the normal, osteogenic (FGF+), and osteogenic (FGF-) MSCs were incubated in 48-well plates for 7 d (10 d after MSC isolation), total RNA was extracted using a QIAshredder and RNeasy kit (Qiagen, Valencia, CA, USA). DNase treatment was performed using RQ1 RNase-free DNase (Promega). The first strand cDNA was synthesised from $1 \mu \mathrm{g}$ of RNA using a SuperScript III Reverse Transcriptase (Invitrogen). The expression levels were determined for ALP, osteopontin (OPN), osteocalcin (OCN), osterix (Osx), and glyceraldehyde3-phosphate dehydrogenase (GAPDH) by real-time TaqMan polymerase chain reaction (PCR) analysis. Optimal oligonucleotide primers and TaqMan probes were designed with ProbeFinder (Roche Diagnostics, Basel, Switzerland) software using rat ALP, OPN, OCN, Osx, and GAPDH sequences from the GenBank database. The primer sets used for PCR were as follows: ALP, 5'-CCGAGTGGTGGTCACGAT-3' and 5' - A C GA G G T C A C G T C C AT C C T-3'; 
OPN, 5' - GAGTTTGGCAGCTCAGAGGA-3' and 5' - TCTGCT TC TGA GATGGGTCA-3'; OCN, 5' - ATAGACTCCGGCGCTACC TC-3' and 5' - C CAGGGGATCTGGGTAGG-3'; Osx, 5' - TGCCCCAACTGTCAGGAG-3' and 5'-GAtGTGGCGGCTGTGAAT-3'; and GAPDH, 5' - T G G G A A G C T G G T C A T C A A C - 3' and 5'-GCATCACCCCATTTGATGTT-3'. TaqMan probes for the tested genes were labelled with carboxyfluorescein (FAM) fluorochrome with the following sequences: ALP, 5'-GGATGCTG-3' (Universal ProbeLibrary probe \# 89); OPN, 5'-GGCTGAAG-3' (Universal ProbeLibrary probe \# 41); OCN, 5'-CTTGGAGC-3' (Universal ProbeLibrary probe \# 125); Osx, 5'-CTGGGCTG-3' (Universal ProbeLibrary probe \# 26); and GAPDH, 5'-CATCACCA-3' (Universal ProbeLibrary probe \# 9). TaqMan PCR was carried out in a $20 \mu \mathrm{L}$ final volume containing $200 \mathrm{nM}$ each of forward and reverse primers, $100 \mathrm{nM}$ Universal ProbeLibrary probe, and a cDNA template. The cycling conditions were $95{ }^{\circ} \mathrm{C}$ for $10 \mathrm{~s}, 60$ ${ }^{\circ} \mathrm{C}$ for $30 \mathrm{~s}$, and $72{ }^{\circ} \mathrm{C}$ for $1 \mathrm{~s}$ for 35 cycles. Real-time TaqMan PCR was performed using a LightCycler 1.5 (Roche Applied Science, Mannheim, Germany). All PCR reactions were performed in duplicate. The gene expression levels of ALP, OPN, OCN, and Osx were quantified as relative expression after normalization to the GAPDH expression level in the same reaction.

\section{MSC seeding on OCP/Col or collagen disks}

Osteogenic (FGF+) MSCs or normal MSCs were used for seeding on $\mathrm{OCP} / \mathrm{Col}$ or collagen disks (Fig. 1BI and 1BII). The $\mathrm{OCP} / \mathrm{Col}$ or collagen disks were placed in 24well polystyrene plates, which inhibited cell attachment on the surface (Ultra Low Attachment Polystyrene; Corning Costar, Acton, MA, USA). After the third passage of the cells, which was almost $10 \mathrm{~d}$ after MSC isolation, normal MSCs or osteogenic (FGF+) MSCs were seeded onto OCP/ Col or collagen disks at a cell density of $2 \times 10^{5}$ cells/disk/ well in $500 \mu \mathrm{L}$ of osteogenic differentiation medium. The 24-well plates containing the seeded disks were incubated on a rotary shaker (Multi Shaker MMA-210; Eyela, Tokyo, Japan) at a speed of $200 \mathrm{rpm}$ for $6 \mathrm{~h}$. The medium in each well was then changed to remove non-adherent cells. OCP/ Col with normal MSCs (OCP/Col-nor-MSCs), OCP/Col, and collagen disks with osteogenic (FGF+) MSCs (OCP/ $\mathrm{Col} \cdot \mathrm{MSCs}$ and $\mathrm{Col} \cdot \mathrm{MSCs})$ were incubated at $37{ }^{\circ} \mathrm{C}$ in a $5 \% \mathrm{CO}_{2}$ environment for $1 \mathrm{~d}$. The preliminary study showed that the cell number one day after seeding $2.0 \times$ $10^{5} \mathrm{MSCs}$ on $\mathrm{OCP} / \mathrm{Col}$ and $\mathrm{Col}$ disks was approximately $78,055 \pm 5,701$ and $69,944 \pm 6,234$, respectively. There was no significant difference between them. Therefore, we concluded that the effects of MSC seeding on bone regeneration could be comparatively analysed between these groups.

\section{Implantation of OCP/Col·n-MSCs, OCP/Col-MSCs, Col·MSCs, OCP/Col, or collagen disks in a rat calvaria bone defect}

Twelve-week-old male Wistar rats (SLC, Hamamatsu, Shizuoka, Japan) were used for this experiment. The principles of laboratory animal care as well as national laws were followed for the use of animals. All procedures were approved by the Animal Research Committee of Tohoku University.

The experimental rats were anaesthetised with an intraperitoneal (i.p.) injection of sodium pentobarbital (50 $\mathrm{mg} / \mathrm{kg}$ ) supplemented with ether inhalation. A calvaria bone defect with a $9 \mathrm{~mm}$ diameter was created as previously described (Kamakura et al., 2006; Kawai et al., 2009). The OCP/Col·nor-MSCs, OCP/Col·MSCs, and Col·MSCs disks that had been cultured for $1 \mathrm{~d}$ were implanted into the trephine defect. As a control, OCP/Col or collagen was implanted in the same manner. As a negative control, untreated animals were processed using the same method, but no disk was implanted in the bone defect. After the defects were treated, the ablated periosteum and the skin were repositioned and sutured, respectively. After 4 weeks, 5 rats in the $\mathrm{OCP} / \mathrm{Col} \cdot$ nor-MSCs group were euthanised and then fixed as described below. In addition, 5 rats each from the $\mathrm{OCP} / \mathrm{Col} \cdot \mathrm{MSC}$, $\mathrm{Col} \cdot \mathrm{MSC}$, $\mathrm{OCP} / \mathrm{Col}$, collagen, and untreated groups were euthanised and fixed at 4 and 8 wks after implantation.

\section{Radiographic analysis}

The rats were anaesthetised with an i.p. injection of sodium pentobarbital $(50 \mathrm{mg} / \mathrm{kg})$ supplemented with ether inhalation. The rats were sacrificed and then the implants were resected together with the surrounding bones. The tissues were immersed in $4 \%$ paraformaldehyde in 0.1 M PBS (pH 7.4) for the tissue fixation and kept overnight at $4{ }^{\circ} \mathrm{C}$. The specimens were radiographed by means of a microradiography unit (Softex CMR Unit; Softex, Tokyo, Japan) with X-ray film (FR; Fuji Photo Film, Tokyo, Japan) under standardised conditions $(20 \mathrm{kV}, 5 \mathrm{~mA}, 1 \mathrm{~min})$, in which OCP showed no radiopacity.

\section{Analysis of bone microstructure}

The calvaria bone was analysed using a Microfocus X-Ray CT System (Scan Xmate-E090; Comscantecno, Kanagawa, Japan). The bone specimens were scanned continuously at increments of $20 \mu \mathrm{m}$ for 512 slices with a tube voltage of $90 \mathrm{kV}$ and a tube current of $0.1 \mathrm{~mA}$. The voxel size was $20 \times 20 \times 20 \mu \mathrm{m}^{3}$. After the scanning, the image data were transferred to a workstation, and the structural indices and the mineralisation of bone were calculated using a threedimensional (3D) image analysis system (TRI/3D-Bon; Ratoc System Engineering, Tokyo, Japan). The TRI/3DBON system builds 3D models from serial tomographic datasets for observation and morphometric analysis. The $3 \mathrm{D}$ images were segmented into voxels identified as bone and marrow. The gray-scale images were segmented using a strict filter to remove noise and a fixed threshold to extract the mineralised bone phase. The isolated small particles in the marrow space and the isolated small holes in the bone were removed using a cluster-labelling algorithm. The bone surface (BS) area was calculated using surface triangulation of the binary data based on the marching cubes method. The bone volume (BV) was calculated using tetrahedrons that corresponded to the enclosed volume of the triangulated surface. The total tissue volume (TV) was the entire volume of the analysis. The normalised index, bone surface-to-volume ratio (BS/BV), and bone 
volume fraction $(\mathrm{BV} / \mathrm{TV})$ were then calculated from these values. In addition to bone structure, we also quantified the bone mineral density (BMD) at the level of calcified bone tissue. In advance, cylindrical phantoms containing an HA slurry with solids and various concentrations of the solution were imaged, and the linear relationship between the HA concentrations $\left(0.3-0.7 \mathrm{~g} / \mathrm{cm}^{3}\right)$ and absorption was verified. The BMD was calibrated using this relationship.

\section{Tissue preparation}

After the radiographic and micro-CT analyses, the samples were decalcified in $10 \%$ ethylenediaminetetraacetic acid in $0.01 \mathrm{M}$ phosphate buffer ( $\mathrm{pH}$ 7.4) for 2-4 weeks at 4 ${ }^{\circ} \mathrm{C}$. The samples were then dehydrated in a graded series of ethanol and embedded in paraffin. The centre of the defect was extracted and sectioned coronally at a thickness of $5 \mu \mathrm{m}$. The sections were stained with haematoxylin and eosin $(\mathrm{H} \& \mathrm{E})$ and the photographs were taken with a photomicroscope (Leica DFC300 FX, Leica Microsystems Japan, Tokyo, Japan).

\section{Quantitative micrograph analysis}

Light micrographs of the sections stained with H\&E were used for histomorphometric measurements. Photographs projecting the overall defect were taken for each specimen. The percentage of newly formed bone in the defect (newBone \%) was calculated as the area of newly formed bone per area of the defect that was originally created by trephination $\times 100$. Similarly, the percentage of remaining OCP in the defect (rem-Imp\%) was calculated as the area of remaining OCP per area of the defect that was originally created by trephination $\times 100$. The new-Bone $\%$ and remImp \% were quantified on a computer using Scion Image public domain software (Scion Corporation, Frederick, MD, USA).

\section{Statistical analysis}

Results are expressed as mean \pm standard deviation (SD). All cellular experiments were performed at least three times and showed reliable reproducibility. Statistical analyses were performed for all of the cellular and histomorphometric experiments using commercial software (Ekuseru-Toukei 2006, Social Survey Research Information, Tokyo, Japan). One-way analysis of variance (ANOVA) was used to compare the means among groups. If the ANOVA was significant, then Tukey's multiple comparison analysis was used as a post-hoc test.

\section{Results}

\section{MSC proliferation and differentiation}

Fig. 2a shows the proliferation of normal, osteogenic (FGF+), and osteogenic (FGF-) MSCs. Six days after MSC isolation, the number of MSCs in each culture condition was almost the same. However, at $10 \mathrm{~d}$ after MSC isolation, the number of osteogenic (FGF+) MSCs was significantly higher than the normal and osteogenic (FGF-) MSCs. The number of osteogenic (FGF-) MSCs was lower than normal MSCs throughout the culture period. There was a significant difference in MSC proliferation between $\mathrm{FGF}(+)$ and $\mathrm{FGF}(-)$ cultures and $\mathrm{FGF}(+)$ and normal cultures on days 10 and 17 of the culture period $(p<0.01)$. The use of osteogenic medium increased the ALP activity of MSCs during the entire observation period regardless of the presence or absence of bFGF (Fig. 2b). Ten days after MSC isolation, osteogenic (FGF+) MSCs exhibited the highest level of ALP activity, which was 1.9 and 64.3 times greater than the osteogenic (FGF-) and normal MSCs, respectively. There was a significant difference in ALP activity of the MSCs between each condition at

Table 1. Results of bone mineral density (BMD) and new-Bone $\%$ in $\mathrm{OCP} / \mathrm{Col}$ with osteogenic $(\mathrm{FGF}+) \mathrm{MSCs}(\mathrm{OCP} / \mathrm{Col} \cdot \mathrm{MSCs})$ or normal MSCs (OCP/Col-nor-MSCs) ay 4 weeks after implantation in rat calvaria defect. ${ }^{*} p<0.05$.

\begin{tabular}{ccc}
\hline & OCP/Col•MSCs & OCP/Col•nor-MSCs \\
\hline BMD (mg/cm $\left.{ }^{3}\right)$ & $693.0( \pm 50.1) *$ & $560.4( \pm 81.9)$ \\
new-Bone (\%) & $44.08( \pm 1.74) *$ & $30.29( \pm 2.70)$ \\
\hline
\end{tabular}

Table 2. Quantification of the bone microstructure in $\mathrm{OCP} /$ $\mathrm{Col} \bullet \mathrm{MSCs}$ and $\mathrm{OCP} / \mathrm{Col}$ at 8 weeks after implantation. ${ }^{*} p$ $<0.05$. TV, total tissue volume; BV, bone volume; BS, bone surface; $\mathrm{BS} / \mathrm{BV}$, bone surface to volume ratio; $\mathrm{BV} / \mathrm{TV}$, bone volume fraction; BMD, bone mineral density.

\begin{tabular}{|c|c|c|}
\hline & OCP/Col•MSCs & OCP/Col \\
\hline $\mathrm{TV}\left(\mathrm{mm}^{3}\right)$ & $45.76 \pm 10.77$ & $39.85 \pm 8.09$ \\
\hline $\mathrm{BV}\left(\mathrm{mm}^{3}\right)$ & $26.80 \pm 11.82$ & $23.42 \pm 6.58$ \\
\hline BS $\left(\mathbf{m m}^{2}\right)$ & $309.09 \pm 58.22$ & $259.34 \pm 40.17$ \\
\hline BS/BV (1/mm) & $12.41 \pm 2.99$ & $11.33 \pm 1.48$ \\
\hline BV/TV (\%) & $56.70 \pm 11.83$ & $58.26 \pm 5.41$ \\
\hline BMD $\left(\mathrm{mg} / \mathrm{cm}^{3}\right)$ & $657.33 \pm 18.16^{*}$ & $632.70 \pm 3.89$ \\
\hline
\end{tabular}



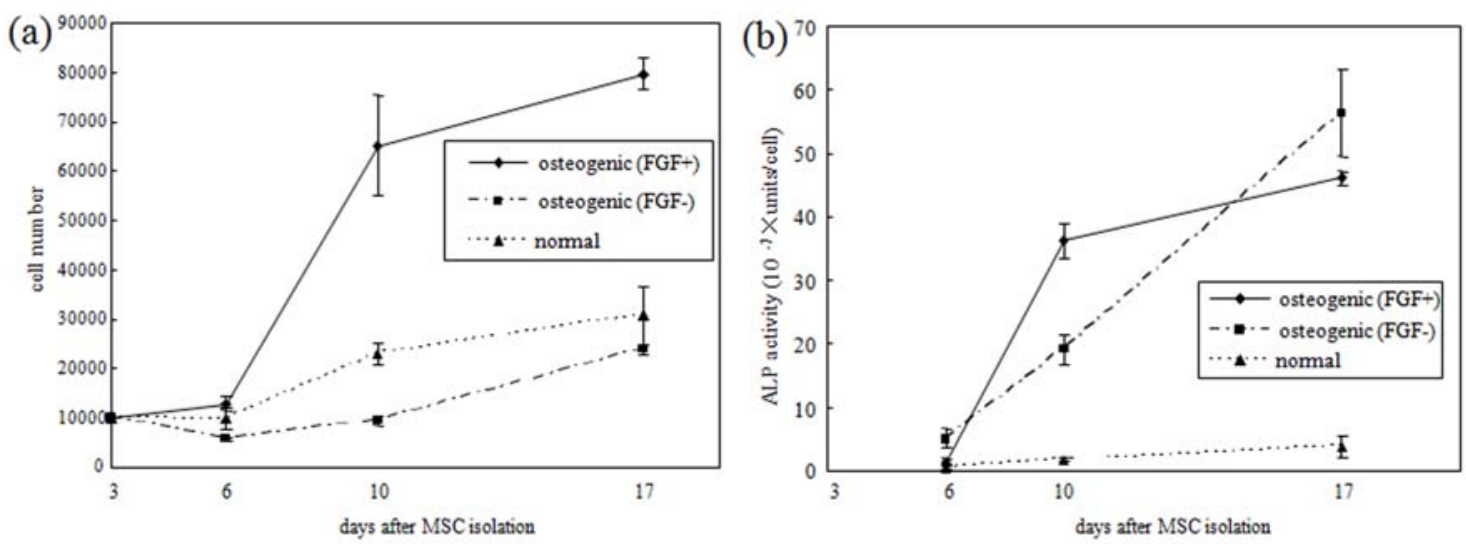

(c) 6 days 10 days 17 days after MSC isolation

osteogenic $(\mathrm{FGF}+)$
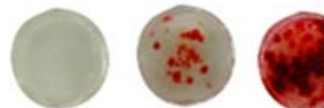

osteogenic (FGF - )
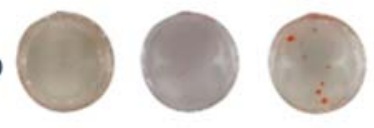

normal
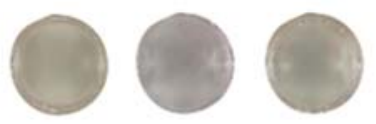

(d)
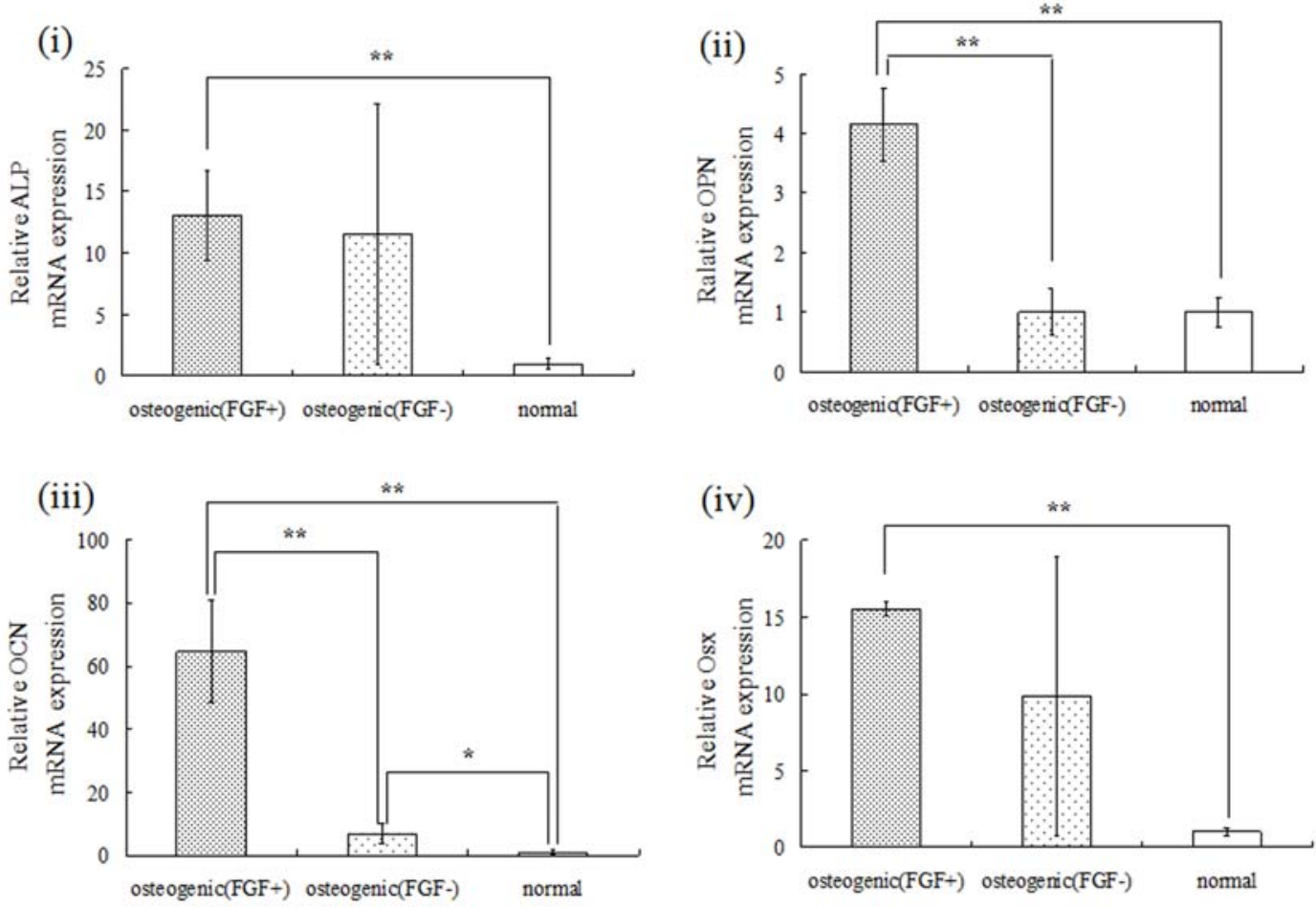

Fig. 2. (a) Results of MSC proliferation after isolation in each culture medium. (b) Results of ALP activity of MSCs after isolation in each culture medium. (c) Alizarin red staining of MSCs cultured in each medium at 6, 10 and 17 $\mathrm{d}$ after isolation. (d) Normalised (i) ALP, (ii) OPN, (iii) OCN, and (iv) Osx mRNA expression (to GAPDH) at $10 \mathrm{~d}$ after MSC isolation by TaqMan real-time PCR. ${ }^{*} p<0.05, * * p<0.01$. 
$10 \mathrm{~d}(p<0.01)$. The ALP activity of FGF(-) MSCs was higher than the $\mathrm{FGF}(+) \mathrm{MSCs}$ on day 17. In addition, there was a significant difference in ALP activity of the MSCs between the $\mathrm{FGF}(+)$ and $\mathrm{FGF}(-)$ groups $(p<0.05)$ and between the $\mathrm{FGF}(+)$ and normal culture groups on day $17(p<0.01)$. The effect of osteogenic $(\mathrm{FGF}+)$ medium on the development of mineralised structures in MSC cultures is shown in Fig. 2c. Alizarin red S staining was used as an indicator of calcification. The addition of bFGF to the osteogenic medium markedly enhanced the amount of mineralised bone-like tissue formation. No calcareous deposit was observed in normal MSCs through $17 \mathrm{~d}$ of the culture period. Fig. 2 d shows the expression of osteogenic genes quantified by real-time TaqMan PCR of osteogenic (FGF+), osteogenic (FGF-), and normal MSCs $10 \mathrm{~d}$ after MSC isolation. The expression levels of ALP, OPN, OCN, and Osx were approximately 13.1, 4.1, 64.5 and 15.5-fold higher, respectively, than those of normal MSCs when the cells were grown in osteogenic (FGF+) medium. The expression levels of OPN and OCN in osteogenic (FGF-) MSCs were significantly lower than those of osteogenic (FGF+) MSCs.

\section{In vivo bone-formation ability of osteogenic (FGF+) MSCs and normal MSCs}

We next examined the process of bone repair by micro$\mathrm{CT}$ in critical-sized defects produced in rat calvaria. Table 1 shows the BMD estimated by micro-CT analysis and the new-Bone $\%$ estimated by histomorphometric measurements of the $\mathrm{OCP} / \mathrm{Col} \cdot \mathrm{MSC}$ s or $\mathrm{OCP} / \mathrm{Col} \cdot$ norMSCs 4 weeks after implantation. Both the BMD and new-Bone $\%$ of $\mathrm{OCP} / \mathrm{Col} \cdot \mathrm{MSC}$ s were significantly higher than those of OCP/Col-nor-MSCs.

\section{Bone regeneration by the OCP/Col scaffold seeded with osteogenic (FGF+) MSCs}

The results of the in vitro and in vivo studies described above demonstrate the significant superiority of the osteogenic $(\mathrm{FGF}+)$ condition over the other culture conditions. Therefore, osteogenic (FGF+) MSCs were used to analyse bone regeneration in combination with the $\mathrm{OCP} / \mathrm{Col}$ scaffold in a rat calvaria defect in order to evaluate whether the crystals of OCP in a collagen matrix can stimulate the introduced MSCs. The results of the radiographic examination are shown in Fig. 3. It has been previously shown that soft $\mathrm{x}$-ray conditions used for measurement do not exhibit any radiopacity in OCP/ Col disks before implantation (Kamakura et al., 2006). $\mathrm{OCP} / \mathrm{Col} \cdot \mathrm{MSCs}$ and $\mathrm{OCP} / \mathrm{Col}$ disks implanted in a rat calvaria defect showed radiopacity at 4 and 8 weeks postimplantation. Col·MSCs or collagen disks implanted in the defect also showed isolated, round radiopacities scattered throughout the defect. There was slight radiopacity along the defect margin in the untreated group. Table 2 shows the quantification of the bone microstructure in the $\mathrm{OCP} /$ $\mathrm{Col} \cdot \mathrm{MSCs}$ and $\mathrm{OCP} / \mathrm{Col}$ disks 8 weeks post-implantation. There were no significant differences in radiomorphometric parameters (BS/BV and BV/TV) between the OCP/ $\mathrm{Col} \cdot \mathrm{MSCs}$ and $\mathrm{OCP} / \mathrm{Col}$ groups. However, the BS of the $\mathrm{OCP} / \mathrm{Col} \cdot \mathrm{MSCs}$ group was greater than the $\mathrm{OCP} / \mathrm{Col}$ group, and the BMD of the $\mathrm{OCP} / \mathrm{Col} \cdot \mathrm{MSCs}$ group was

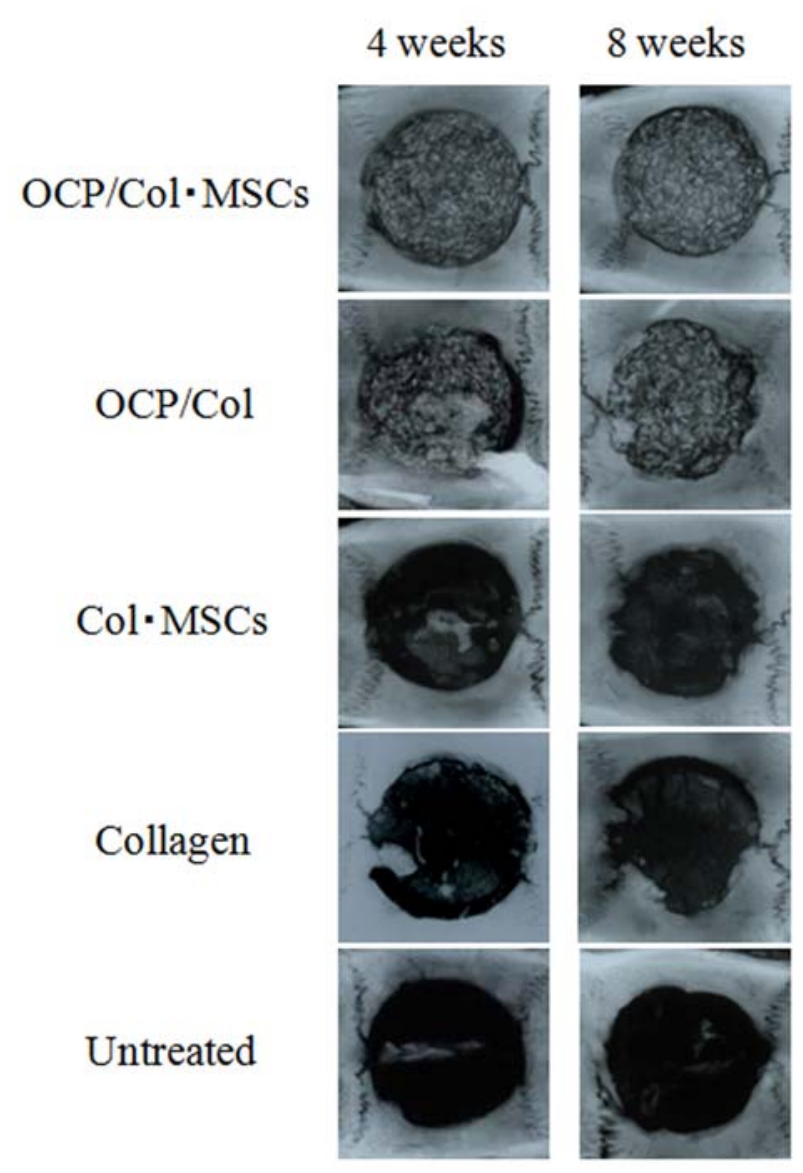

Fig. 3. Radiographs of rat calvaria defect treated with $\mathrm{OCP} / \mathrm{Col} \cdot \mathrm{MSCs}, \mathrm{OCP} / \mathrm{Col}, \mathrm{Col} \cdot \mathrm{MSCs}$, or collagen at 4 and 8 weeks. Defect size was $9 \mathrm{~mm}$ in diameter.

significantly higher than the OCP/Col group. Figs. 4 and 5 show the histological sections of each material in the presence or absence of cultured MSCs implanted in a rat calvaria defect. At 4 weeks, newly formed bone was clearly observed in the connective tissue of the defect implanted with a $\mathrm{OCP} / \mathrm{Col} \cdot \mathrm{MSCs}$ disk. In contrast, newly formed bone was limited to the area within the defect for rats implanted with an $\mathrm{OCP} / \mathrm{Col}$ disk. Newly formed bone was minimal in the defect implanted with $\mathrm{Col} \cdot \mathrm{MSC}$ or collagen disks as well as the group left untreated. At 8 weeks, a greater expansion of newly formed bone was observed in the calvaria defect implanted with $\mathrm{OCP} / \mathrm{Col} \cdot \mathrm{MSC}$ or $\mathrm{OCP} /$ $\mathrm{Col}$ disks. Some OCP granules within the OCP/Col disk were surrounded by multinucleated giant cells (MNGCs), but inflammatory cell infiltration was not observed at the implantation site. In the Col·MSCs and collagen groups, bone formation was observed sporadically in the collagen disks. In addition, bone regeneration in the untreated control was restricted to the defect margin.

Histomorphometric findings for the new-Bone $\%$ and rem-Imp \% are shown in Figs. 6 and 7, respectively. The values for new-Bone $\% \pm \mathrm{SD}$ in the $\mathrm{OCP} / \mathrm{Col} \cdot \mathrm{MSC}, \mathrm{OCP} /$ $\mathrm{Col}$, Col·MSC, collagen, and untreated groups were 44.1 $\pm 1.7,22.7 \pm 1.3,16.6 \pm 0.9,10.8 \pm 1.5$ and $4.1 \pm 0.8$ at 4 weeks, and $52.6 \pm 1.3,39.7 \pm 1.6,21.2 \pm 2.8,16.6 \pm 1.2$ and $4.2 \pm 3.7$ at 8 weeks, respectively. The values for rem- 


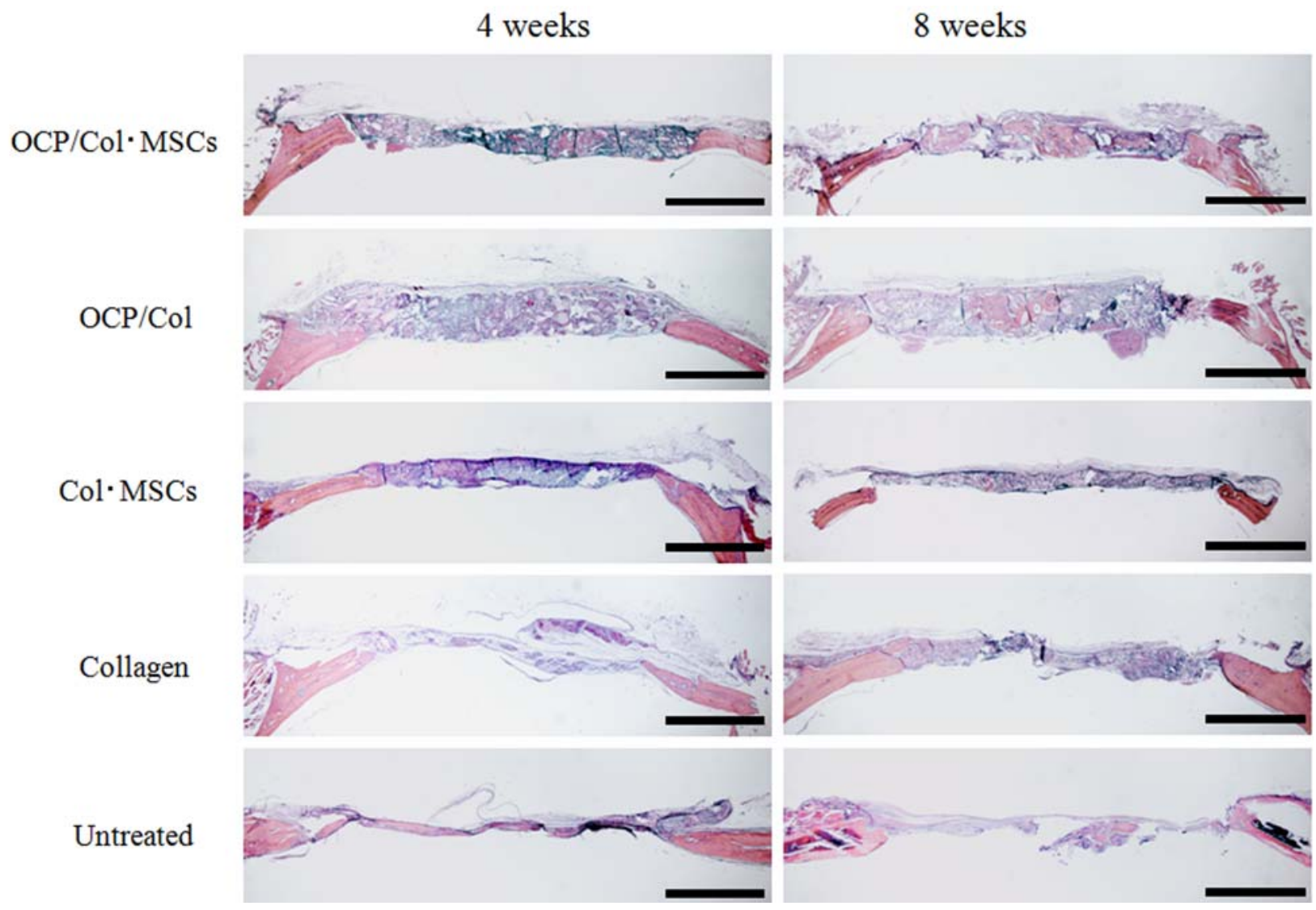

Fig. 4. Overview of histological sections with haematoxylin-eosin staining on calvaria defect treated with OCP/ $\mathrm{Col} \cdot \mathrm{MSCs}, \mathrm{OCP} / \mathrm{Col}, \mathrm{Col} \cdot \mathrm{MSCs}$, or collagen at 4 and 8 weeks. Bars $=2 \mathrm{~mm}$.

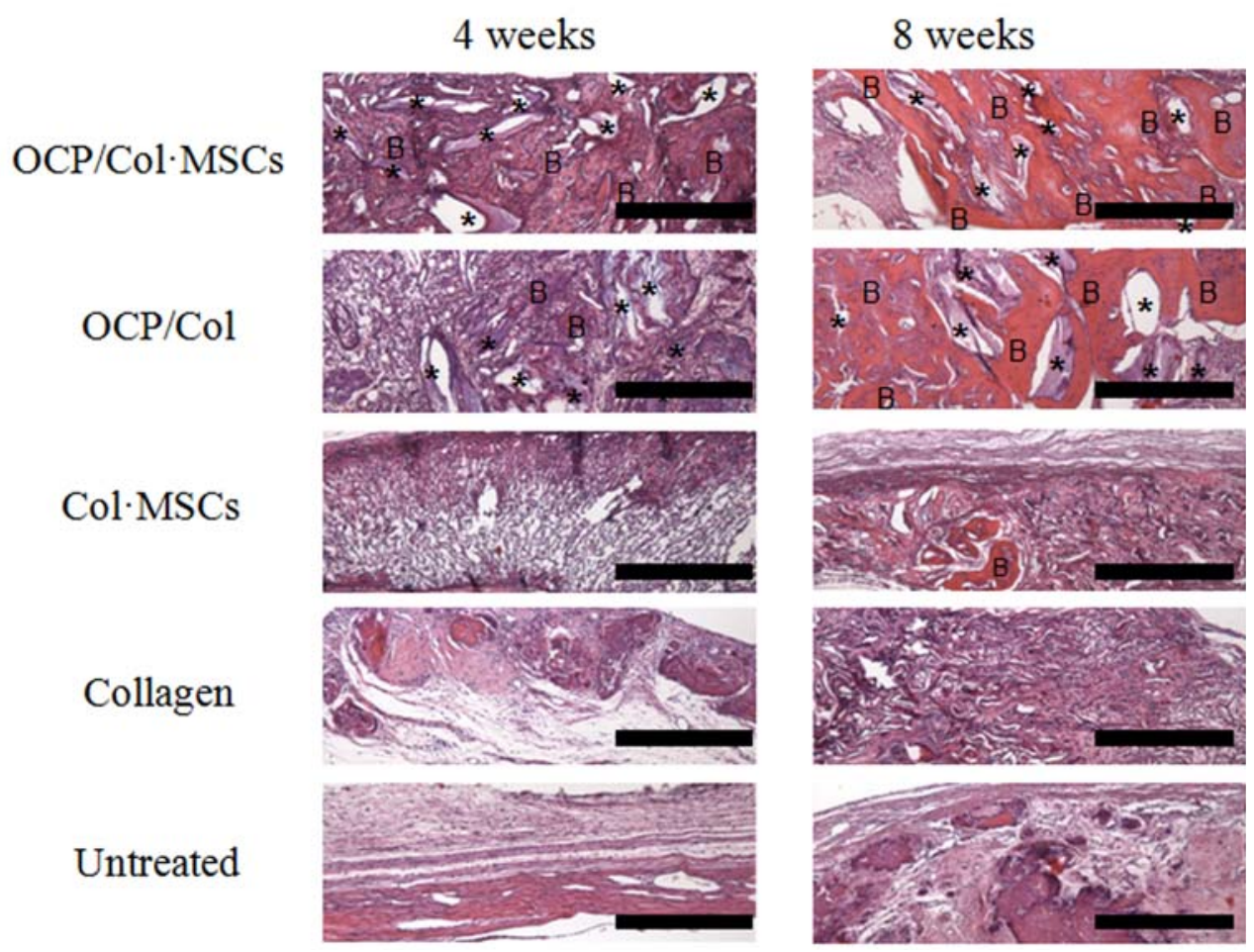

Fig. 5. Histological examination of calvarial defects with implantation of $\mathrm{OCP} / \mathrm{Col} \cdot \mathrm{MSCs}$, $\mathrm{OCP} / \mathrm{Col}$, Col·MSCs, or collagen. Bars $=500 \mu \mathrm{m} . *=$ remaining OCP. $\mathrm{B}=$ newly formed bone. 


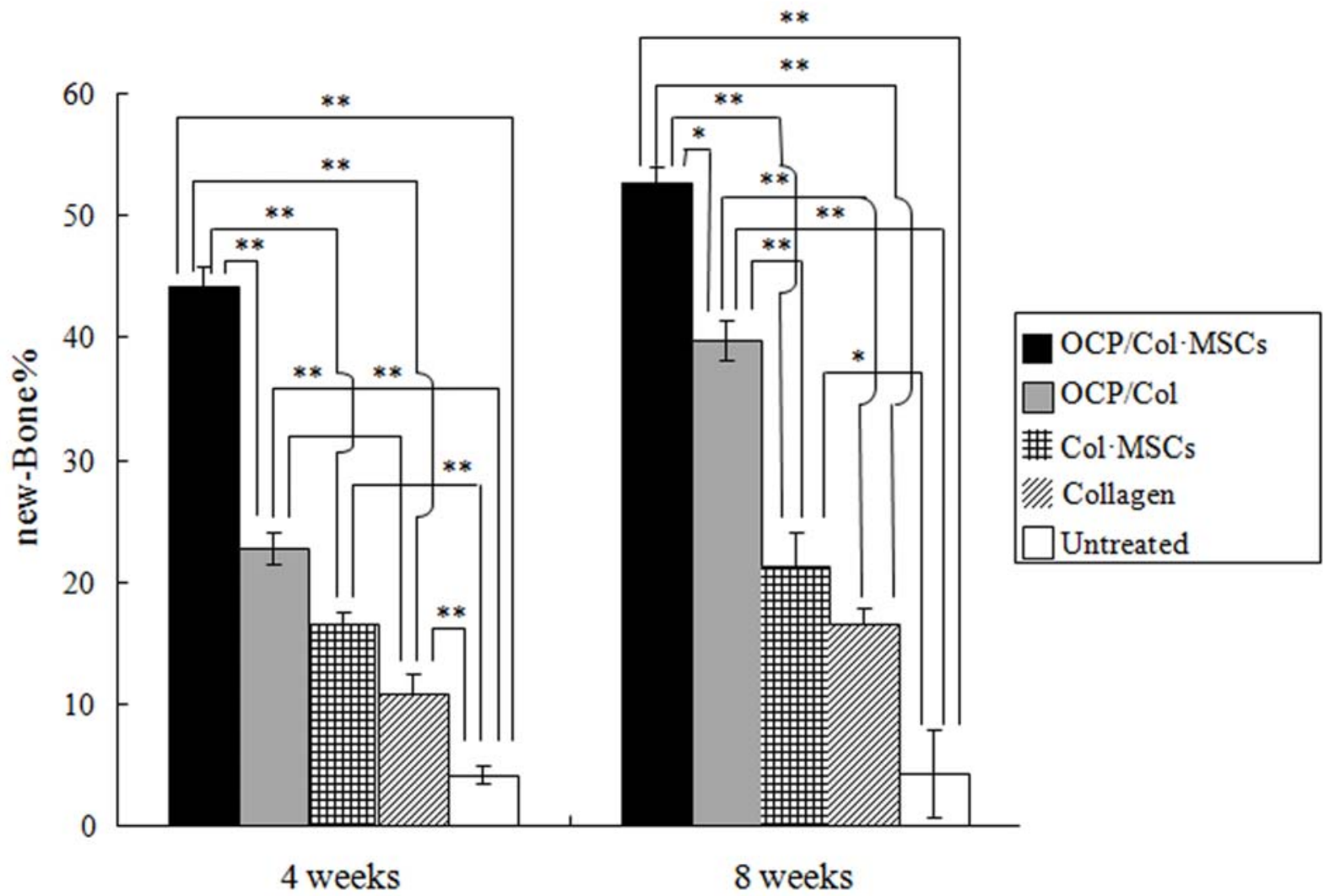

Fig. 6. Histomorphometric findings regarding the area of newly formed bone (new-Bone $\%$ ) in the defects. ${ }^{*} p<$ $0.05, * * p<0.01$.

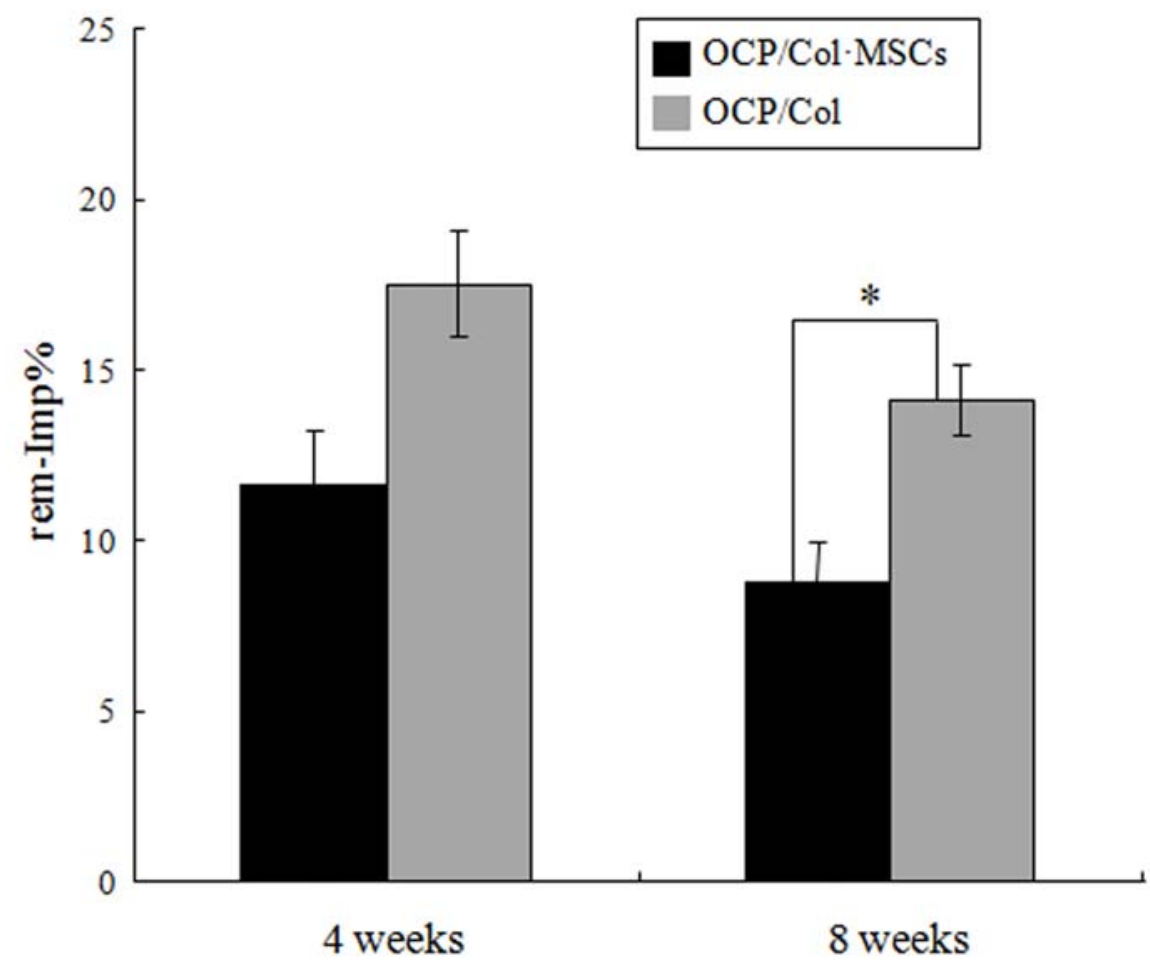

Fig. 7. Quantitative analysis of remaining implant OCP granules (rem-Imp\%) in the defect. ${ }^{*} p<0.05$. 
$\mathrm{Imp} \% \pm \mathrm{SD}$ in the $\mathrm{OCP} / \mathrm{Col} \cdot \mathrm{MSC}$ and $\mathrm{OCP} / \mathrm{Col}$ groups were $11.6 \pm 1.6$ and $17.5 \pm 1.6$ at 4 weeks, and $8.9 \pm 1.1$ and $14.1 \pm 0.0$ at 8 weeks, respectively. The new-Bone $\%$ of the $\mathrm{OCP} / \mathrm{Col} \cdot \mathrm{MSC}$ group was significantly higher than the $\mathrm{OCP} / \mathrm{Col}$ group. Moreover, the new-Bone $\%$ of the OCP/ $\mathrm{Col} \cdot \mathrm{MSC}$ and $\mathrm{OCP} / \mathrm{Col}$ groups significantly increased at 8 weeks. The analysis revealed that the OCP in the OCP/ $\mathrm{Col} \cdot \mathrm{MSC}$ group was resorbed significantly more than that of the $\mathrm{OCP} / \mathrm{Col}$ group. In the $\mathrm{OCP} / \mathrm{Col} \cdot \mathrm{MSC}$ and $\mathrm{Col} \cdot \mathrm{MSC}$ groups, there was a significant difference in new-Bone $\%$ throughout the implantation period. In addition, the value for the new-Bone $\%$ of the Col·MSC group was slightly higher than the collagen alone group throughout the implantation period.

\section{Discussion}

MSCs have been considered to be a suitable autologous cell source for the generation of tissue-engineered bone. Defining the culture conditions for the in vitro proliferation and differentiation of MSCs is important for tissue engineering therapy. Several reports have shown that bFGF markedly increases the growth rate and lifespan of MSCs. Furthermore, the incubation of MSCs with bFGF retains their differentiation potential throughout numerous mitotic divisions (Tutsumi et al., 2001; Bianchi et al., 2003). In these reports, bFGF was added at the first passage and continued until the cells were confluent. When the cells reached confluence, differentiation was induced by osteogenic medium containing dexamethasone, ascorbic acid and $\beta$-glycerophosphate without bFGF. In our present study, we used this osteogenic culture condition as a "normal" condition (Fig. 1). We showed that osteogenic medium containing bFGF (osteogenic $(\mathrm{FGF}+)$ ) enhanced the proliferation of MSCs when added early in the culture period (Fig. 1), but also increased the activity of ALP, the expression level of osteogenic differentiation related genes, and the amount of mineralised nodule formation (Fig. 2). Pitaru et al. previously showed that bFGF has the capacity to stimulate both the growth and differentiation of rat MSCs (Kotev-Emeth et al., 2002; Pitaru et al., 1993). Scutt et al. also reported that bFGF could stimulate the osteogenic differentiation of rat MSCs, and that continued exposure to bFGF increased the formation of bone-like tissue in vitro (Scutt and Bertram, 1999). Our results are consistent with these reports. In this study, we observed very weak alizarin red staining in osteogenic (FGF-) MSCs with increasing ALP activity by day 17 of the culture period (Figs. $2 b$ and $2 \mathrm{c}$ ). There are several possible explanations for these findings, such as (1) the cell proliferation in the absence of FGF was slow, whereas that in the presence of FGF was markedly enhanced; (2) ALP activity was expressed as a value per unit cell; or (3) the lower cellular density in the absence of FGF resulted in the detection of very few mineral deposits. Similar findings have also been reported in a previous study (Maegawa et al., 2007).

We further examined the in vivo bone-forming efficiency of MSCs cultured in osteogenic (bFGF+) medium or normal medium using $\mathrm{OCP} / \mathrm{Col}$ scaffolds. Enhanced new bone formation and a higher bone mineral density were observed with the transplantation of MSCs that had been cultured in osteogenic $(\mathrm{FGF}+)$ medium (Table 1). Yoshikawa et al. previously reported that in vitro ALP activity and bone Gla protein content correlated well with the amount of newly formed bone in vivo (Yoshikawa et al., 1992). In fact, the present procedure using osteogenic medium containing bFGF increased ALP activity compared to normal culture conditions, which resulted in enhanced formation of new bone.

It has been reported that several weeks are required to prepare and differentiate MSCs to osteoblastic cells for transplantation (i.e., primary culture in standard medium for $10 \mathrm{~d}$ followed by a subculture in differentiation medium for 2 weeks (Yoshikawa et al., 1996; Okamoto et al., 2006)). In contrast, the present procedure shortened the time required for transplantation to approximately $10 \mathrm{~d}$. These results show the usefulness of our in vitro cell culture expansion-differentiation technique using an osteogenic (FGF+) condition, which was confirmed after in vivo implantation. Since shortening the culture period for osteogenic differentiation reduces the time required for the bone defect to heal, the present procedure used to accelerate both the growth and biochemical functions of MSCs could be therapeutically useful. However, it remains to be shown that the present material would have a similar effect on human MSCs.

Several studies have suggested that inorganic ceramic materials not only act as simple scaffold materials, but also affect osteoblast functions on the cell surface through direct interactions (Kokubo et al., 2009). A recent study showed that $\beta$-TCP can affect the signal transduction of human primary osteoblasts (Lu and Zreiqat, 2010). Dissolution of the material elements, such as calcium ions, and the adsorption of biomolecules on their surfaces explain the bioactivity of these ceramics (Kokubo, 1991; Ducheyne and Qui, 1999). $\beta$-TCP has also been shown to affect MSC functions, including protein synthesis (Kasten et al., 2008), bone induction within a gelatin sponge matrix in relation to its dose (Takahashi et al., 2005), and the osteogenic ability after chondrogenic differentiation (Janicki et al., 2010). $\mathrm{BCP}$ and Ca-deficient HA reduced MSC differentiation and the calcification ability when added directly to the cell culture media (Saldaña et al., 2009). Ca-deficient HA was also found to induce a stronger inhibition of MSC differentiation than $\mathrm{BCP}$ when the cells were indirectly co-cultured with these materials, which was explained by calcium uptake from the medium due to the crystal imperfection of Ca-deficient HA (Saldaña et al., 2009). Therefore, calcium phosphate ceramics may affect MSC differentiation.

We previously showed that $\mathrm{OCP} / \mathrm{Col}$ disks induce bone regeneration better than collagen disks (Kamakura et al., 2006; Kawai et al., 2009). Interestingly, in the present study we found that $\mathrm{OCP} / \mathrm{Col}$ disks implanted without MSCs into bone defects enhanced bone regeneration better than collagen disks with MSCs (Figs. 3-6). However, micro$\mathrm{CT}$ quantification demonstrated significant differences in bone mineral density between the $\mathrm{OCP} / \mathrm{Col}$ composites implanted with and without MSCs, suggesting that MSCs inoculated on the $\mathrm{OCP} / \mathrm{Col}$ composite were effective at inducing bone maturation (Table 2). In fact, the amount 
of newly formed bone was higher in the $\mathrm{OCP} / \mathrm{Col} \cdot \mathrm{MSCs}$ group than the Col-MSCs group. These results suggest that the inclusion of OCP crystals in this composite material supports both host cells and transplanted MSCs for the induction of new bone formation. Recent studies have demonstrated that OCP is capable of inducing the differentiation of stromal cells into osteoblastic cells in vitro (Shelton et al., 2006; Liu et al., 2007; Anada et al., 2008). It has been suggested that the stimulatory capacity of OCP to raise osteoconductive characteristics stems from a physicochemical change caused by the conversion of OCP to HA (Suzuki et al., 2006a; Suzuki et al., 2006b). Even with the OCP/Col composite, OCP crystals converted to apatite under physiological conditions (Suzuki et al., 2009). The OCP-HA conversion of OCP crystals is accompanied by calcium ion consumption as well as phosphate ion release into the surrounding solution (Suzuki et al., 2006a), and it has been hypothesised that these ions can modify osteoblast functions (Bellows et al., 1992; Dvorak et al., 2004). In fact, the corresponding changes in ion concentration have also been observed in culture medium incubated with OCP (Anada et al., 2008; Takami et al., 2009), suggesting that the solution-mediated stimulation of osteoblasts occurs not only under in vitro physiological conditions, but also after in vivo implantation.

The amount of OCP remaining 8 weeks after implantation was significantly different between the groups with and without MSCs (Fig. 7). Previous in vivo studies have shown that OCP increases the number of osteoclast-like cells that are positive for tartrate-resistant acid phosphatase activity (Imaizumi et al., 2006; Kikawa et al., 2009; Murakami et al., 2010). These results suggest that OCP itself enhances bone formation, which is accompanied by biodegradation and a concomitant increase in osteoclastic cellular resorption of OCP (Imaizumi et al., 2006; Kikawa et al., 2009; Murakami et al., 2010). We previously reported (Takami et al., 2009) that OCP can induce the formation of osteoclasts from precursors in bone marrow cells if co-cultured with osteoblasts, even in the absence of vitamin $D_{3}$, which is a factor that increases the osteoclast-inducing molecule receptor activator of NF- $\mathrm{kB}$ ligand (RANKL) in osteoblasts. Therefore, it should be determined whether OCP has a similar effect on MSCs for inducing osteoclast differentiation.

\section{Conclusions}

The results of the present study demonstrate that OCP crystals in a collagen matrix are able to support exogenous MSC-mediated bone regeneration in an $\mathrm{OCP} / \mathrm{Col}$ scaffold implanted in rats with critical-sized defects. These results also suggest that OCP crystals have a similar effect on host osteoblasts that invade the $\mathrm{OCP} / \mathrm{Col}$ scaffold within the implantation site. The MSCs grown using the present cell culture procedure with osteogenic $(\mathrm{FGF}+)$ medium accelerated the growth and differentiation of the cells more efficiently, and may provide a useful cellular source for bone regeneration if combined with the $\mathrm{OCP} / \mathrm{Col}$ scaffold. Taken together, our results show that $\mathrm{OCP} / \mathrm{Col} \cdot \mathrm{MSCs}$ disks have osteogenic capabilities that are supported by the physicochemical properties of the OCP structure and are therefore potential candidates for autologous transplantation.

\section{Acknowledgements}

This study was partially supported by Grants-in-Aid (17076001, 19390490, 20659304, 21591910, 23390450, 23659999, and 23106010) from the Ministry of Education, Science, Sports, and Culture of Japan.

\section{References}

Anada T, Kumagai T, Honda Y, Masuda T, Kamijo R, Kamakura S, Yoshihara N, Kuriyagawa T, Shimauchi H, Suzuki O (2008) Dose-dependent opsteogenic effect of octacalcium phosphate on mouse bone marrow stromal cells. Tissue Eng 14: 965-978.

Bellows CG, Heersche JN, Aubin JE (1992) Inorganic phosphate added exogenously or released from betaglycerophosphate initiates mineralization of osteoid nodules in vitro. Bone Miner 17: 15-29.

Bianchi G, Banfi A, Mastrogiacomo M, Notaro R, Luzzatto L, Cancedda R, Quarto R (2003) Ex vivo enrichment of mesenchymal cell progenitors by fibroblast growth factor 2. Exp Cell Res 287: 98-105.

Brown WE, Smith JP, Lehr JR, Frazier AW (1962) Crystallographic and chemical relations between octacalcium phosphate and hydroxyapatite. Nature 196: 1050-1055.

Brown WE, Mathew M, Tung MS (1981) Crystal chemistry of octacalcium phosphate. Prog Cryst Growth Charact 4: 59-87.

Bucholz RW (2002) Nonallograft osteoconductive bone graft substitutes. Clin Orthop 395: 44-52.

Chevallier N, Anagnostou F, Zilber S, Bodivit G, Maurin S, Barrault A, Bierling P, Hernigou P, Layroll P, Rouard H (2010) Osteoblastic differentiation of human mesenchymal stem cells with platelet lysate. Biomaterials 31: 270-278.

Chow L (2001) Solubility of calcium phosphates. In: Octacalcium Phosphate (Chow LC, Eanes ED, eds), Karger, Basel, pp. 94-111.

Dennis JE, Esterly K, Awadallah A, Parrish CR, Poynter GM, Goltry KL (2007) Clinical-scale expansion of a mixed population of bone marrow-derived stem and progenitor cells for potential Use in Bone Tissue Regeneration. Stem Cells 25: 2575-2582.

Driessens FC, van Dijk JW, Borggreven JM (1978) Biological calcium phosphates and their role in the physiology of bone and dental tissues I. Composition and solubility of calcium phosphates. Calcif Tissue Res 26: 127-137.

Ducheyne P, Qui Q (1999) Bioactive ceramics: The effect of surface reactivity on bone formation and bone cell function. Biomaterials 20: 2287-2303.

Dvorak MM, Siddiqua A, Ward DT, Carter DH, Dallas SL, Nemeth EF, Riccardi D (2004) Physiological 
changes in extracellular calcium concentration directly control osteoblast function in the absence of calciotropic hormones. Proc Natl Acad Sci USA 101: 5140-5145.

Eidelman N, Chow LC, Brown WE (1987) Calcium phosphate saturation levels in ultrafiltered serum. Calcif Tissue Int 40: 71-78.

Espitalier F, Vinatier C, Lerouxel E, Guicheux J, Pilet P, Moreau F, Daculsi G, Weiss P, Malard O (2009) A comparison between bone reconstruction following the use of mesenchymal stem cells and total bone marrow in association with calcium phosphate scaffold in irradiated bone. Biomaterials 30: 763-769.

Fowler BO, Moreno EC, Brown WE (1966) Infra-red spectra of hydroxyapatite, octacalcium phosphate and pyrolysed octacalcium phosphate. Arch Oral Biol 11: 477 492.

Grundel RE, Chapman MW, Yee T, Moore DC (1991) Autogeneic bone marrow and porous biphasic calcium phosphate ceramic for segmental bone defects in the canine ulna. Clin Orthop Relat Res 266: 244-258.

Imaizumi H, Sakurai M, Kashimoto O, Kikawa T, Suzuki O (2006) Comparative study on osteoconductivity by synthetic octacalcium phosphate and sinterd hydroxyapatite in rabbit bone marrow. Calcif Tissue Int 78: 45-54.

Janicki P, Kasten P, Kleinschmidt K, Luginbuehl R, Richter W (2010) Chondrogenic pre-induction of human mesenchymal stem cells on $\beta$-TCP: enhanced bone quality by endochondral heterotopic bone formation. Acta Biomater 6: 3292-3301.

Kamakura S, Sasano Y, Homma H, Suzuki O, Kagayama M, Motegi K (1999) Implantation of octacalcium phosphate (OCP) in rat skull defects enhances bone repair. J Dent Res 78: 1682-1687.

Kamakura S, Sasaki K, Honda Y, Anada T, Suzuki O (2006) Octacalcium phosphate combined with collagen orthotopically enhances bone regeneration. J Biomed Mater Res B Appl Biomater 79: 210-217.

Kasten P, Vogel J, Luginbuhl R, Niemeyer P, Tonak M, Lorenz H, Helbig L, Weiss, Fellenberg J, Leo A, Simank H, Richter W (2005) Ectopic bone formation associated with mesenchymal stem cells in a resorbable calcium deficient hydroxyapatite carrier. Biomaterials 26: 5879-5889.

Kasten P, Beyen I, Niemeyer P, Luginbühl R, Bohner M, Richter W (2008) Porosity and pore size of $\beta$-tricalcium phosphate scaffold can influence protein production and osteogenic differentiation of human mesenchymal stem cells: an in vitro and in vivo study. Acta Biomater 4: 19041915.

Kawai T, Anada T, Honda Y, Kamakura S, Matsui K, Matsui A, Sasaki K, Morimoto S, Echigo E, Suzuki O (2009) Synthetic octacalcium phosphate augments bone regeneration correlated with its content in collagen scaffold. Tissue Eng PartA 15: 23-32.

Kawate K, Yajima H, Ohgushi H, Kotobuki N, Sugimoto K, Ohmura T, Kobata Y, Shigematsu K, Kawamura K, Tamai K, Takakura Y (2006) Tissue-engineered approach for the treatment of steroid-induced osteonecrosis of the femoral head: Transplantation of autologous mesenchymal stem cells cultured with $\beta$-tricalcium phosphate ceramics and free vascularized fibula. Artif Organs 30: 960-962.

Kikawa T, Kahimoto O, Imaizumi H, Kokubun S, Suzuki O (2009) Intramembranous bone tissue response to biodegradable octacalcium phosphate implant. Acta Biomater 5: 1756-1766

Kokubo T (1991) Bioactive glass ceramics: Properties and applications. Biomaterials 12: 155-163.

Kokubo T, Matsushita T, Takadama H, Kizuki T (2009) Development of bioactive materials based on surface chemistry. J Euro Ceramic Soc 29: 1267-1274.

Kotev-Emeth S, Pitaru S, Pri-Chen S, N S (2002) Establishment of a rat long-term culture expressing the osteogenic phenotype: dependence on dexamethasone and FGF-2. Connc Tissue Res 43: 606-612.

Kuznetsov SA, Huang KE, Marshall GW, Robey PG, Mankani MH (2008) Long-term stable canine mandibular augmentation using autologous bone marrow stromal cells and hydroxyapatite/Ttricalcium phosphate. Biomaterials 29: 4211-4216.

LeGeros RZ (2002) Properties of osteoconductive biomaterials: calcium phosphates. Clin Orthop 72: 81-98.

Liu Y, Cooper PR, Barralet JE, Shelton RM (2007) Influence of calcium phosphate crystal assemblies on the proliferation and osteogenic gene expression of rat bone marrow stromal cells. Biomaterials 28: 1393-1403.

Lu Z, Zreiqat H (2010) Beta-tricalcium phosphate exerts osteoconductivity through $\alpha 2 \beta 1$ integrin and downstream MAPK/ERK signaling pathway. Biochem Biophys Res Commun 394: 323-329.

Maegawa N, Kawamura K, Hirose M, Yajima H, Takakura Y, Ohgushi H (2007) Enhancement of osteoblastic differentiation of mesenchymal stromal cells cultured by selective combination of bone morphogenetic protein-2 (BMP-2) and fibroblast growth factor-2 (FGF-2). J Tissue Eng Regen Med 1: 306-313.

Matsui A, Anada T, Masuda T, Honda Y, Miyatake N, Kawai T, Kamakura S, Echigo S, Suzuki O (2010) Mechanical stress-related calvaria bone augmentation by onlayed octacalcium phosphate-collagen implant. Tissue Eng Part A 16: 139-151.

Miyatake N, Kishimoto KN, Anada T, Imaizumi H, Itoi E, Suzuki O (2009) Effect of partial hydrolysis of octacalcium phosphate on its osteoconductive characteristics. Biomaterials 30: 1005-1014.

Murakami Y, Honda Y, Anada T, Shimauchi H, Suzuki O (2010) Comparative study on bone regeneration by synthetic octacalcium phosphate with various granule sizes. Acta Biomater 6: 1542-1548.

Nelson DG, McLean JD (1984) High-resolution electron microscopy of octacalcium phosphate and its hydrolysis products. Calcif Tissue Int 36: 219-232.

Okamoto M, Dohi Y, Ohgushi H, Shimaoka H, Ikeuchi M, Matsushima A, Yonemasu K, Hosoi H (2006) Influence of the porosity of hydroxyapatite ceramics on in vitro and in vivo bone formation by cultured rat bone marrow stromal cells. J Mater Sci: Mater Med 17: 327-336.

Pitaru S, Kotev-emeth S, Noff D, Kaffulaer S, Savion N (1993) Effect of basic fibroblast growth factor on the 
growth and differentiation of adult stromal bone marrow cells: Enhanced development of mineralized bone-like tissue in culture. J Bone Mineral Res 8: 919-929.

Quarto R, Mastrogiacomo M, Cancedda R, Kutepov SM, Mukhachev V, Lavroukov A, Kon E, Marcacci M (2001) Repair of large bone defects with the use of autologous bone Marrow stromal cells. N Engl J Med 344: 385-386.

Saldaña L, Sánchez-Salcedo S, Izquierdo-Barba I, Bensiamar F, Munuera L, Vallet-Regí M, Vilaboa N (2009) Calcium phosphate-based particles influence osteogenic maturation of human mesenchymal stem cells. Acta Biomater 5: 1294-1305.

Scutt A, Bertram P (1999) Basic fibroblast growth factor in the presence of dexamethasone stimulates colony formation, expansion, and osteoblastic differentiation by rat Bone Marrow stromal cells. Calcif Tissue Int 64: 69-77.

Shelton RM, Liu Y, Cooper PR, Gbureck U, German MJ, Barralet JE (2006) Bone marrow cell gene expression and tissue construct assembly using octacalcium phosphate microscaffolds. Biomaterials 27: 2874-2881.

Sugihara F, Mandai Y, Yoshikawa M, Toda T (1995) Experimental-study of octacalcium phosphate for bone substitute material. J Dent Res 74: 973-973.

Sugihara F, Oonishi H, Minamigawa K, Maidai Y, Tsuji E, Yoshikawa M, Toda T (1996) Bone tissue reaction of octacalcium phosphate-collagen conjugated sponge. Bioceramics 9: 399-402.

Suzuki O, Nakamura M, Miyasaka Y, Kagayama M, Sakurai M (1991) Bone formation on synthetic precursors of hydroxyapatite. Tohoku J Exp Med 164: 37-50.

Suzuki O, Nakamura M, Miyasaka Y, Kagayama M, Sakurai M (1993) Maclura pomifera agglutinin-binding glycoconjugates on converted apatite from synthetic octacalcium phosphate implanted into subperiosteal region of mouse calvaria. Bone Miner 20: 151-166.

Suzuki O, Yagishita H, Amano T, Aoba T (1995) Reversible structural changes of octacalcium phosphate and labile acid phosphate. J Dent Res 74: 1764-1769.

Suzuki O, Kamakura S, Katagiri T (2006a) Surface chemistry and biological responses to synthetic octacalcium phosphate. J Biomed Mater Res Appl Biomater 77B: 201212.

Suzuki O, Kamakura S, Katagiri T, Nakamura M, Zhao B, Honda Y, Kamijo R (2006b) Bone formation enhanced implanted octacalcium phosphate involving conversion into Ca-deficient hydroxyapatite. Biomaterials 27: 2671 2681.

Suzuki Y, Kamakura S, Honda Y, Anada T, Hatori K, Sasaki K, Suzuki O (2009) Appositional bone formation by OCP-collagen composite. J Dent Res 88: 1107-1112.

Takahashi Y, Yamamoto M, Tabata Y (2005) Enhanced osteoinduction by controlled release of bone morphogenetic protein-2 from biodegradable sponge composed of gelatin and $\beta$-tricalcium phosphate. Biomaterials 26: 4856-4865.

Takami M, Mochizuki A, Yamada A, Tachi K, Zhao B, Miyamoto Y, Anada T, Honda Y, Nakamura M, Suzuki O, Kamijo R (2009) Osteoclast differentiation induced by synthetic octacalcium phosphate through rankl expression in osteoblasts. Tissue Eng PartA 15: 3991-4000.

Tutsumi S, Shimazu A, Miyazaki K, Pan H, Koike C, Yoshida E, Takagishi K, Kato Y (2001) Retention of multilineage differentiation potential of mesenchymal cells during proliferation in response to FGF. Biochem Biophys Res Commun 288: 413-419.

Werntz JR, Lane JM, Burstein AH, Justin R, Klein R, Tomin E (1996) Qualitative and quantitative analysis of orthotopic bone regeneration by marrow. J Orthop Res 14: 85-93.

Winand L (1965) Physico-chemical study of some apatitic calcium phosphates. In: Tooth Enamel (Stack MV, Fearnhead RS, eds), John Wright and Sons, Bristol, pp 15-19.

Yoshikawa T, Ohgushi H, Okumura M, Tamai S, Dohi Y, Moriyama T (1992) Biochemical and histlogical sequences of membranous ossification in ectopic site. Calcif Tissue Int 50: 184-188.

Yoshikawa T, Ohgushi H, Tamai S (1996) Immediate bone forming capability of prefabricated osteogenic hydroxyapatite. J Biomed Mater Res 32: 481-492.

Yoshikawa T, Ohgushi H, Ichijima K, Takakura Y (2004) Bone regeneration by grafting of cultured human bone. Tissue Eng 10: 688-697.

\section{Discussion with Reviewers}

Reviewer I: As mentioned in the Introduction section, OCP tends to convert to HA in a proper aqueous environment. Did this conversion occur during the in vitro culture of MSCs and biomaterials? Did this conversion occur in the in vivo experiments? If the answer is yes to either question, is it possible that the better bone generation on $\mathrm{OCP} / \mathrm{Col}$ could be attributed to this conversion (i.e., attributed to $\mathrm{HA}$, not to $\mathrm{OCP})$ ?

Authors: We did not analyse the OCP-HA conversion in vitro and in vivo in the present study. However, the previous studies confirmed that OCP without any matrix materials and OCP in Col matrix or in Alginate matrix tend to convert to HA phases in the cell culture media with or without osteoblastic cells and also in vivo if implanted in rat calvaria defects (Suzuki et al., 2006b, text reference) and in mouse (Suzuki et al., 1991, text reference) and rat (Suzuki et al., 2009, text reference) subperiosteal region on calvaria and in mouse abdomen subcutaneous tissue near facia (Suzuki et al., 1993). The previous report (Suzuki et al., 2006b) showed that bone regeneration determined histomorphometrically and osteoblastic differentiation at mRNA level in semi-quantitative (Suzuki et al., 2006b) and quantitative (Anada et al., 2008, text reference) analyses are enhanced by OCP which is hydrolysing into HA in situ whereas they are not enhanced by OCP hydrolysate (Cadeficient HA material obtained via hydrolysis experiment of OCP). From these results, we concluded that a process from OCP to HA itself, not OCP crystals or HA crystals, should exhibit a stimulatory effect on osteoblastic differentiation and bone regeneration. 